\title{
A Novel Mutation in FOXC1 in a Lebanese Family with Congenital Heart Disease and Anterior Segment Dysgenesis: Potential Roles for NFATC1 and DPT in the Phenotypic Variations
}

OPEN ACCESS

Edited by:

David Hassel,

Heidelberg University, Germany

Reviewed by:

Steven Clive Greenway,

University of Calgary, Canada

Yuqi Zhao,

University of California,

Los Angeles,

United States

${ }^{*}$ Correspondence:

Fadi Bitar

fadi.bitar@aub.edu.lb;

Georges Nemer

georges.nemer@aub.edu.lb

Specialty section:

This article was submitted to Cardiovascular Genetics and

Systems Medicine,

a section of the journal

Frontiers in Cardiovascular Medicine

Received: 17 July 2017 Accepted: 31 August 2017 Published: 20 September 2017

Citation:

Khalil A, Al-Haddad C, Hariri H,

Shibbani K, Bitar F, Kurban M,

Nemer G and Arabi M (2017) A Novel

Mutation in FOXC1 in a Lebanese

Family with Congenital Heart Disease

and Anterior Segment Dysgenesis:

Potential Roles for NFATC1 and

DPT in the Phenotypic Variations.

Front. Cardiovasc. Med. 4:58.

doi: 10.3389/fcvm.2017.00058

\begin{abstract}
Athar Khalil', Christiane Al-Haddad ${ }^{2}$, Hadla Hariri', Kamel Shibbani ${ }^{1}$, Fadi Bitar ${ }^{3 *}$, Mazen Kurban ${ }^{1,4,5}$, Georges Nemer ${ }^{1 *}$ and Mariam Arabi ${ }^{3}$

${ }^{1}$ Department of Biochemistry and Molecular Genetics, American University of Beirut, Beirut, Lebanon, ${ }^{2}$ Department of Ophthalmology, American University of Beirut, Beirut, Lebanon, ${ }^{3}$ Department of Pediatrics and Adolescent Medicine, American University of Beirut, Beirut, Lebanon, ${ }^{4}$ Department of Dermatology, American University of Beirut, Beirut, Lebanon, ${ }^{5}$ Department of Dermatology, Columbia University, New York, NY, United States
\end{abstract}

Congenital heart diseases (CHDs) are still the leading cause of death in neonates. Anterior segment dysgenesis is a broad clinical phenotype that affects the normal development of the eye, leading in most of the cases to glaucoma which is still a major cause of blindness for children and adolescents. Despite tremendous insights gained from genetic studies, a clear genotype-phenotype correlation is still difficult to draw. In Lebanon, a small country with still a high rate of consanguineous marriages, there are little data on the epidemiology of glaucoma amongst children with or without CHD. We carried out whole exome sequencing (WES) on a family with anterior segment dysgenesis, and $\mathrm{CHD}$ composed of three affected children with glaucoma, two of them with structural cardiac defects and three healthy siblings. The results unravel a novel mutation in FOXC1 (p. R127H) segregating with the phenotype and inherited from the mother, who did not develop glaucoma. We propose a digenic model for glaucoma in this family by combining the FOXC1 variant with a missense variant inherited from the father in the dermatopontin (DPT) gene. We also unravel a novel NFATC1 missense mutation predicted to be deleterious and present only in the patient with a severe ocular and cardiac phenotype. This is the first report on FOXC1 using WES to genetically characterize a family with both ocular and cardiac malformations. Our results support the usage of such technology to have a better genotype-phenotype picture for Mendelian-inherited diseases for which expressivity and penetrance are still not answered.

Keywords: anterior segment dysgenesis, congenital heart disease, forkhead box c1, digenic, whole exome sequencing

\section{INTRODUCTION}

Glaucoma is the second leading cause of blindness in the world, according to the World Health Organization $(1,2)$. It is characterized by a progressive damage of the eye's optic nerve, which is highly influenced by fluid building up in the front part of the eye leading to an increase in the intraocular pressure $(\mathrm{IOP})(3,4)$. Genetic predisposition, aging, and environmental factors do play essential roles 
in the developing of the disease in adults; however, the financial, social, and familial burden is more sensed in the congenital and juvenile cases $(3,5-8)$. These cases are mostly linked to developmental defects in the formation and differentiation of the cells that make the ocular system in general, and in particular the optic nerve and the anterior segment which comprise the iris, the lens, and the cornea $(3,5,6)$.

Pediatric glaucomas are divided into two major types: primary congenital glaucoma (PCG), which accounts for $50-70 \%$ of all childhood cases, and syndromic-associated glaucomas that include among others juvenile open angle glaucoma (JOAG) (OMIM\#37750, \#603383, and \#137760), aniridia (OMIM\#106210), and the Axenfeld-Rieger syndrome (ARS) (OMIM\#180500,\#601499, and \#602482). Linkage analyses and genome wide association studies have identified many loci and genes implicated in PCG, mostly inherited in an autosomal recessive form, diagnosed in the first year of life, and largely prevalent in countries with high consanguinity. Among these, MYOC (myocilin) and CYP1B1 (cytochrome P450) represent the frequently mutated genes linked to the phenotype $(6,9)$. Interestingly, a digenic mode of inheritance with both CYP1B1 and MYOC mutations has been documented in patients with PCG highlighting the potential involvement of common genetic and molecular players in the disease (10). JOAG is inherited mainly as a dominant trait with an onset age ranging from 3 to 35 years and characterized by high IOP requiring in most of the cases early surgical treatment. The major genetic players are mutations in $M Y O C$ with high prevalence and penetrance, followed by OPTN (optineurin) and WDR36 (WD repeat containing protein 36) (11). Aniridia is a very rare panocular disease whereby glaucoma is diagnosed in $50-70 \%$ of the cases at later ages (end of adolescent, early adulthood). Mostly inherited as autosomal dominant, it is mainly caused by mutations in PAX6 (paired-box gene 6), the master regulator gene of eye development $(12,13)$. As for ARS, it is an autosomal dominant disorder characterized by iris stromal hypoplasia, prominent Schwalbe line (embryotoxon), adhesion between the iris and Schwalbe line, microcornea, corneal opacity, and increased IOP that leads to glaucoma in about $50 \%$ of the cases (3, 14-17). Patients with ARS can also have maxillary hypoplasia, dental anomalies, umbilical hernia, and/or hypospadias. More rarely, they may have hydrocephalus, hearing loss, cardiac and kidney abnormalities, and congenital hip dislocation in addition to the ocular abnormalities. Globally, mutations in two genes FOXC1 (forkhead box c1) and PITX2 (paired-like homeodomain transcription factor 2) have been shown to be responsible for most of the cases with complete penetrance but variable expressivity (14, 17-19).

FOXC1 belongs to the Forkhead box (FOX) family of transcription factors, which share an evolutionarily conserved DNA-binding domain known as the Forkhead domain (20-22). In addition to the eye, it plays a dose-dependent evolutionary conserved role in the early development of the blood vessels, the brain, the heart, and the somites (23). Foxc1 homozygous null mutant mice have a lethal phenotype; they die pre- and perinatally with hemorrhagic hydrocephalus and multiple skeletal, ocular, and genitourinary defects. They also suffer from cardiovascular defects, most notably, interruption or coarctation of the aortic arch $(24,25)$. In addition to its critical role during ocular development, Foxc1 has a protective role in the adult eye. It regulates antiapoptotic genes such as Foxola in order to maintain homeostasis in the adult trabecular meshwork (TM), which is constantly exposed to aqueous humor (26). By maintaining programmed cell death and thus proliferation of TM cells, FOXC1 is a major player in the onset of glaucoma once deregulated. In humans, deleterious FOXC1 mutations associated with ARS span the entire region of the protein including the DNA-binding and activation domains, resulting in reduced transcriptional activity due to haploinsufficiency. Despite the in vitro studies that assess these differential activities, a genotype-phenotype correlation map could not be established to explain the variable expressivity of the phenotype even among patients harboring the same mutation.

In Lebanon, a small country with still frequent consanguineous marriages, only one recent study addressed the genetic basis of congenital glaucoma (27). We hereby describe the first FOXC1 missense mutation using whole exome sequencing (WES) in a Lebanese family with anterior segment dysgenesis and cardiac phenotypes. We showed that this novel mutation has different expressivity, but we propose a digenic model of inheritance that includes NFATC1 to explain the severe cardiac phenotype in one of the patients. Our findings explicitly call for a revision of the expressivity and penetrance terms in genetic inheritance by using WES as a tool to explain genotype-phenotype correlations.

\section{MATERIALS AND METHODS}

\section{Patients Recruitment}

The study was approved by the institutional review board at the American University of Beirut. All patients, their legal guardians, and family members signed an informed consent form before being enrolled in the study. Patients presenting to the Department of Pediatrics and Adolescent Medicine with Congenital Heart Disease (CHD) were serially recruited in the study under IRB approved protocol BioCh.Gn.01. Blood samples were collected from a family consisting of six children and their parents. Two children presented with glaucoma and VSD and one with glaucoma only. Standard clinical evaluation included a complete physical examination, electrocardiography and two-dimensional transthoracic echocardiography with color Doppler were obtained. Family consanguinity history was utilized in constructing pedigrees after interviewing all patients and their parents.

\section{Exome Sequencing}

Blood samples were collected from all members, and the DNA was extracted using the Qiagen Blood-Midi kit (Qiagen Science Inc., Germantown, MD, USA), following the manufacturer's protocol. DNA quantification was performed using the NanoDrop (Thermo Fisher Scientific Inc., Waltham, MA, USA) at the molecular core facility at AUB. One microgram of coded DNA samples from both parents, and the six children were shipped to Macrogen (South Korea, www.dna.macrogen.com) where exome sequencing was performed using the V6 Sureselect target 
enrichment capture system from Agilent on a HiSeq4000 platform from Illumina (San Diego, CA, USA).

\section{Sanger Sequencing}

Sanger sequencing was used to confirm the missense mutation in FOXC1 by exome sequencing. Briefly, primers were designed to amplify partially the region on exon 1 of the gene that covers the mutation: 5'-CCTACGGGCCCTACACG-3' (F) and 5'-GTTGTCCACGCTGAAGCC-3' (R). The $749 \mathrm{bp} \mathrm{PCR}$ products were resolved on agarose gels, then purified using the QIAquick kits (Qiagen, Science Inc. Germantown, MD, USA). Sanger sequencing was carried out as previously described on an ABI3500 (Applied Biosystems, Foster City, CA, USA) platform at the American University of Beirut Molecular Core Facility.

\section{Data Analysis}

Primary analysis was done at Macrogen. The Fastq files were mapped to the reference genome using the Burrows-Wheeler Alignment tool. The Genome Analysis Toolkit was used for variants calling, and the SnEff software was used to annotate the variants. The Illumina Variant Studio was used to filter the variants as per their frequency and presence or absence in the affected family members versus the healthy individuals.

\section{RESULTS}

\section{Clinical Evaluation: Is Glaucoma Separate from CHD?}

Index patient II.3 (Figure 1) was referred to the Pediatrics Heart Center at the American University of Beirut Medical Center for consultation at the age of 8 years. Echocardiography showed a small perimembranous ventricular septal defect (VSD) with aneurysmal tissue formation. The color Doppler study was indicative of normal pulmonary arterial pressure with no valvar stenosis. There was no need for cardiac medications, and the parents were advised to a follow-up visit after 2 years. Concomitant with this date, the parents had a newborn girl, patient II.6 who was admitted to the emergency room for congestive heart failure

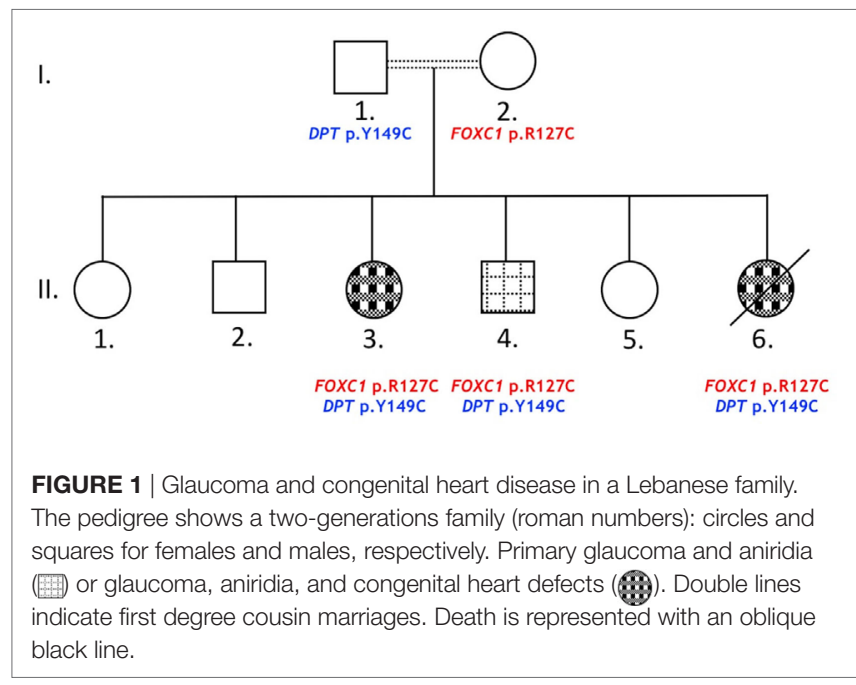

at 4 months of age. Echocardiography results showed a mild biventricular hypertrophy, with a more prominent right ventricle phenotype. A large subaortic VSD was noted with an overriding aorta, and pulmonary atresia reminiscent of a Tetralogy of Fallot (TOF)-like phenotype. The color Doppler imaging showed a trace tricuspid regurgitation, and a patent ductus arteriosus (PDA) like structure arising most likely from the transverse aorta. A rightmodified Blalock-Taussig shunt was performed, and in parallel the patient was diagnosed with aniridia and glaucoma. Her ocular examination at the age of 6 months showed partial aniridia in both eyes with corneal edema on the right and corneal opacification with neovascularization on the left. Examination under anesthesia revealed elevated IOP at $21 \mathrm{mmHg}$ with enlarged corneas measuring $11 \mathrm{~mm}$ on the right and $13 \mathrm{~mm}$ on the left. Dilated fundoscopy showed a normal posterior pole on the right with a cup to disk ratio of 0.4. No view could be obtained of the left fundus. She underwent partial trabeculotomy in the right eye as it was as discovered intraoperatively that she had incomplete development of her Schlemm's canal. This finding prohibited complete trabeculotomy and confirmed an anterior segment dysgenesis diagnosis. Two months later, she underwent Ahmed tube implantation in the right eye due to persistent glaucoma. Her postoperative course was complicated by a right corneal ulcer and an infection of the shunt that necessitated its removal 3 months after placement. She subsequently did well postoperatively with a limited hyphema and was lost to follow-up later. The same ocular finding was also detected in two of her siblings (II.3 and II.4), but was absent from the remaining four. The parents were not examined at our facilities, but they denied having any cardiac or ocular problem. The family was enrolled in our ongoing genetic study on CHDs, but patient II.6 passed away few months later, and no follow-up was made with the rest of the family members.

\section{Genetic Analysis (I): A Novel FOXC1 Missense Variant with Variable Expressivity}

The results of WES yielded an average of 98,521 variants enclosing both single nucleotide polymorphisms and insertions/deletions in the six children and their parents. A first round of variants' filtering consists of keeping only variants from an inclusive list of 65 genes (Table S1 in Supplementary Material) implicated in glaucoma, anterior segment dysgenesis, microcornea, and microphthalmia. We use an arbitrary model of inheritance that includes X-linked recessive, or autosomal dominant, or autosomal recessive with a minor allele frequency (MAF) less than 5\%, and excluding synonymous and in-frame insertions/deletions variants. Only one novel variant in the coding region of FOXC1 was detected in the three affected children (Table 1); the chr 6:1611059C $>\mathrm{T}$ variant leads to a missense mutation p.R127C (NM_001453.2) in the DNA-binding domain of the protein. While being absent from the father and the three non-affected children, this missense mutation is inherited from the healthy mother and is predicted to be deleterious and damaging as ascertained by Sanger sequencing (Figure 2). Additionally, a missense variant in PCMTD1 was encountered in all family members, affected and unaffected, therefore excluding it from being responsible for the ocular phenotype (Table 1). 
TABLE 1 | Variants in genes implicated in glaucoma, anterior segment dysgenesis, microcornea, and microphthalmia in the affected patients.

\begin{tabular}{|c|c|c|c|c|c|c|c|c|c|c|c|c|c|c|c|c|c|c|c|c|}
\hline Sample & Gene & Variant & Coordinate & Chr & Type & Filters & Quality & $\begin{array}{l}\text { Inherited } \\
\text { from }\end{array}$ & $\begin{array}{l}\text { Allelic } \\
\text { depths }\end{array}$ & Transcript & Consequence & $\begin{array}{l}\text { Protein } \\
\text { position }\end{array}$ & $\begin{array}{l}\text { Amino } \\
\text { acids }\end{array}$ & Sift & PolyPhen & dbSNP ID & $\begin{array}{l}\text { Allele freq } \\
\text { global } \\
\text { minor }\end{array}$ & $\begin{array}{l}\text { Allele } \\
\text { Freq } \\
\text { Evs }\end{array}$ & $\begin{array}{c}\text { EVs } \\
\text { Coverage }\end{array}$ & $\begin{array}{c}\text { EVS } \\
\text { Samples }\end{array}$ \\
\hline 11.6 & CNTNAP2 & $\mathrm{C}>\mathrm{C} / \mathrm{CTG}$ & 148106477 & 7 & Insertion & PASS & 658.77 & Father & 21,18 & NM_014141.5 & $\begin{array}{l}\text { splice_region_ } \\
\text { variant, } \\
\text { intron_variant, } \\
\text { feature_elongation }\end{array}$ & 0 & & & & & 0 & 0 & 0 & 0 \\
\hline 11.4 & CNTNAP2 & $\mathrm{G}>\mathrm{G} / \mathrm{T}$ & 146805232 & 7 & Snv & PASS & 36.77 & None & 2,3 & NM_014141.5 & $\begin{array}{l}\text { splice_region_ } \\
\text { variant, } \\
\text { intron_variant }\end{array}$ & 0 & & & & & 0 & 0 & 54 & 6503 \\
\hline 11.6 & COL11A1 & $\mathrm{GA}>\mathrm{G} / \mathrm{G}$ & 103496805 & 1 & Deletion & PASS & 78.03 & Both & 0,4 & NM_080629.2 & $\begin{array}{l}\text { splice_region_ } \\
\text { variant, } \\
\text { intron_variant, } \\
\text { feature_truncation }\end{array}$ & 0 & & & & $\begin{array}{l}\text { rs67059272, } \\
\text { rs36076089 }\end{array}$ & 0 & 0 & 20 & 6498 \\
\hline 11.3 & COL11A1 & $G A>G / G$ & 103496805 & 1 & Deletion & PASS & 52.28 & Both & 0,3 & NM_080629.2 & $\begin{array}{l}\text { splice_region_ } \\
\text { variant, } \\
\text { intron_variant, } \\
\text { feature_truncation }\end{array}$ & 0 & & & & $\begin{array}{l}\text { rs67059272, } \\
\text { rs36076089 }\end{array}$ & 0 & 0 & 20 & 6498 \\
\hline 11.4 & COL11A1 & $\mathrm{GA}>\mathrm{G} / \mathrm{G}$ & 103496805 & 1 & Deletion & PASS & 52.28 & Both & 0,3 & NM_080629.2 & $\begin{array}{l}\text { splice_region_ } \\
\text { variant, } \\
\text { intron_variant, } \\
\text { feature_truncation }\end{array}$ & 0 & & & & $\begin{array}{l}\text { rs67059272, } \\
\text { rs36076089 }\end{array}$ & 0 & 0 & 20 & 6498 \\
\hline 11.6 & FOXC1 & $\mathrm{C}>\mathrm{C} / \mathrm{T}$ & 1611059 & 6 & Snv & PASS & 615.77 & Mother & 28,20 & NM_001453.2 & missense_variant & 127 & $\mathrm{R} / \mathrm{C}$ & deleterious(0) & probably_damaging(1) & & 0 & 0 & 96 & 6503 \\
\hline 11.3 & FOXC1 & $\mathrm{C}>\mathrm{C} / \mathrm{T}$ & 1611059 & 6 & Snv & PASS & 896.77 & Mother & 27,28 & NM_001453.2 & missense_variant & 127 & $\mathrm{R} / \mathrm{C}$ & deleterious(0) & probably_damaging(1) & & 0 & 0 & 96 & 6503 \\
\hline 11.4 & FOXC1 & $\mathrm{C}>\mathrm{C} / \mathrm{T}$ & 1611059 & 6 & Snv & PASS & 704.77 & Mother & 15,22 & NM_001453.2 & missense_variant & 127 & $\mathrm{R} / \mathrm{C}$ & deleterious(0) & probably_damaging(1) & & 0 & 0 & 96 & 6503 \\
\hline 11.6 & GALC & $\mathrm{GA}>\mathrm{G} / \mathrm{G}$ & 88417095 & 14 & Deletion & PASS & 282.1 & Both & 1,12 & NM_000153.3 & $\begin{array}{l}\text { splice_region_ } \\
\text { variant, } \\
\text { intron_variant, } \\
\text { feature_truncation }\end{array}$ & 0 & & & & rs11300320 & 0 & 0 & 11 & 5784 \\
\hline 11.3 & GALC & $\mathrm{GA}>\mathrm{G} / \mathrm{G}$ & 88417095 & 14 & Deletion & PASS & 799.77 & Both & 0,30 & NM_000153.3 & $\begin{array}{l}\text { splice_region_ } \\
\text { variant, } \\
\text { intron_variant, } \\
\text { feature_truncation }\end{array}$ & 0 & & & & rs11300320 & 0 & 0 & 11 & 5784 \\
\hline 11.4 & GALC & $\mathrm{GA}>\mathrm{G} / \mathrm{G}$ & 88417095 & 14 & Deletion & PASS & 477.77 & Both & 0,18 & NM_000153.3 & $\begin{array}{l}\text { splice_region_ } \\
\text { variant, } \\
\text { intron_variant, } \\
\text { feature_truncation }\end{array}$ & 0 & & & & rs11300320 & 0 & 0 & 11 & 5784 \\
\hline 11.6 & PCMTD1 & $A>A / C$ & 52733079 & 8 & Snv & PASS & 76.77 & None & 20,4 & NM_052937.2 & missense_variant & 302 & $S / R$ & tolerated(0.09) & $\begin{array}{l}\text { probably_ } \\
\text { damaging(0.975) }\end{array}$ & rs75865149 & 0 & 0 & 115 & 6503 \\
\hline 11.6 & PCMTD1 & $T>T / G$ & 52733128 & 8 & Snv & PASS & 238.77 & Ambiguous & 15,9 & NM_052937.2 & missense_variant & 286 & $\mathrm{~N} / \mathrm{T}$ & tolerated(0.56) & benign(0.012) & rs62506083 & 0 & 0 & 188 & 6503 \\
\hline 11.3 & PCMTD1 & $T>T / G$ & 52733128 & 8 & Snv & PASS & 81.77 & Ambiguous & 14,5 & NM_052937.2 & missense_variant & 286 & $\mathrm{~N} / \mathrm{T}$ & tolerated(0.56) & benign $(0.012)$ & rs62506083 & 0 & 0 & 188 & 6503 \\
\hline & PCMTD1 & $T>T / G$ & 52733128 & 8 & Snv & PASS & 187.77 & Ambiguous & 19,11 & NM_052937.2 & missense_variant & 286 & $N / T$ & tolerated(0.56) & benign(0.012) & rs62506083 & 0 & 0 & 188 & 6503 \\
\hline
\end{tabular}




\section{Genetic Analysis (II): A DPT Missense Variant Inherited from the Father Exclusively in the Affected Glaucomatous Patients}

In order to understand the absence of ocular manifestations in the mother, we hypothesized that an additional variant inherited from the father with no effect on its own might be needed in conjunction with the FOXC1 variant to cause Glaucoma in the affected children. Using the Illumina variant studio, we filtered the variants that are only shared by the three affected individuals (II.3, 4, and 6) and their father I.1 (Figure 1). The filtering process included all coding variants with an MAF $<5 \%$ and excluded synonymous and in-frame insertions/deletions variants. Among the six variants (Table 2), two are missense variants in the RNA Binding Motif Protein 43 (RBM43) gene (p.V34L) and the dermatopontin (DPT) gene (p.Y149C). The latter is novel, not reported previously in any database, and predicted to be deleterious and damaging (Table 2), while the former is reported in the SNP database, and the RBM43 gene was not shown to be expressed in the eye. On the contrary, previous reports do support a role for $D P T$ in the eye and potentially in glaucoma, thus reinforcing its potential role in the underlying phenotype especially that it is not expressed in the mother. The remaining four variants do not have an effect on the coding sequences or splicing, thus excluding them from any role in the ocular phenotype.

\section{Genetic Analysis (III): A Novel Missense Variant in NFATC1 Responsible for the Severe Cardiac Phenotype?}

To delineate the cardiac phenotype observed exclusively in probands II.3 and II.6, a variant filtering approach was conducted using a two-arm strategy. The variants should have the following characteristics; first, they must have an MAF $<5 \%$, exclusively shared by the two individuals and absent from the others, and second, they must be inherited from both parents (assuming a recessive model of inheritance) or from the father assuming a combinatorial effect with the FOXC1 variant. In the former strategy, only eight variants were shared (Table 3), but none could explain the cardiac phenotype alone or in conjunction with FOXC1 since there are no published data about their role neither in cardiac development or in CHD. In the latter, 18 shared variants were detected with no cardiac relevance for anyone of them; of note a nonsense variant in ZNF28 inherited from the father was not encountered in any database (Table 3). We moved then to assess each individual with cardiac defect alone by using the same strategy above. In patient II.3, 13 variants in a total of 7 genes were inherited from the father, however, none were previously implicated in CHD, nor were they implicated in a FOXC1 partnership (Table 4). In contrast, patient II.6 had 17 variants in 15 genes inherited from her father but not encountered in the other siblings. Two of these variants were in genes implicated in heart development and cardiac pathology, NFATC1 and OBSCN (Table 4). The two variants chr18:77170979 G>A for NFATC1 and chr1:228462101 G>A for OBSCN lead to novel missense variants, $\mathrm{p} . \mathrm{R} 222 \mathrm{Q}$ and $\mathrm{p}$.C1880Y respectively. They were not reported previously and are predicted to be deleterious and damaging, potentially explaining the severe cardiac phenotype in the patient (Table 4).

\section{Genetic Analysis (IV): A Family at Risk of Sudden Death?}

A final thorough analysis of all predicted deleterious variants in the exome sequencing of patient II.6 showed a novel nonsense mutation in desmoglein (DSG2) (Figure 3A), a gene implicated in arrhythmogenic right ventricular dysplasia. The variant is inherited from the father and is shared between four siblings (Figure 3B), including all three affected children with either glaucoma and/or CHD, in addition to a healthy sibling (II.1).

\section{DISCUSSION}

Anterior segment dysgenesis englobes a wild spectrum of ocular defects that include among others the ARS, which is frequently
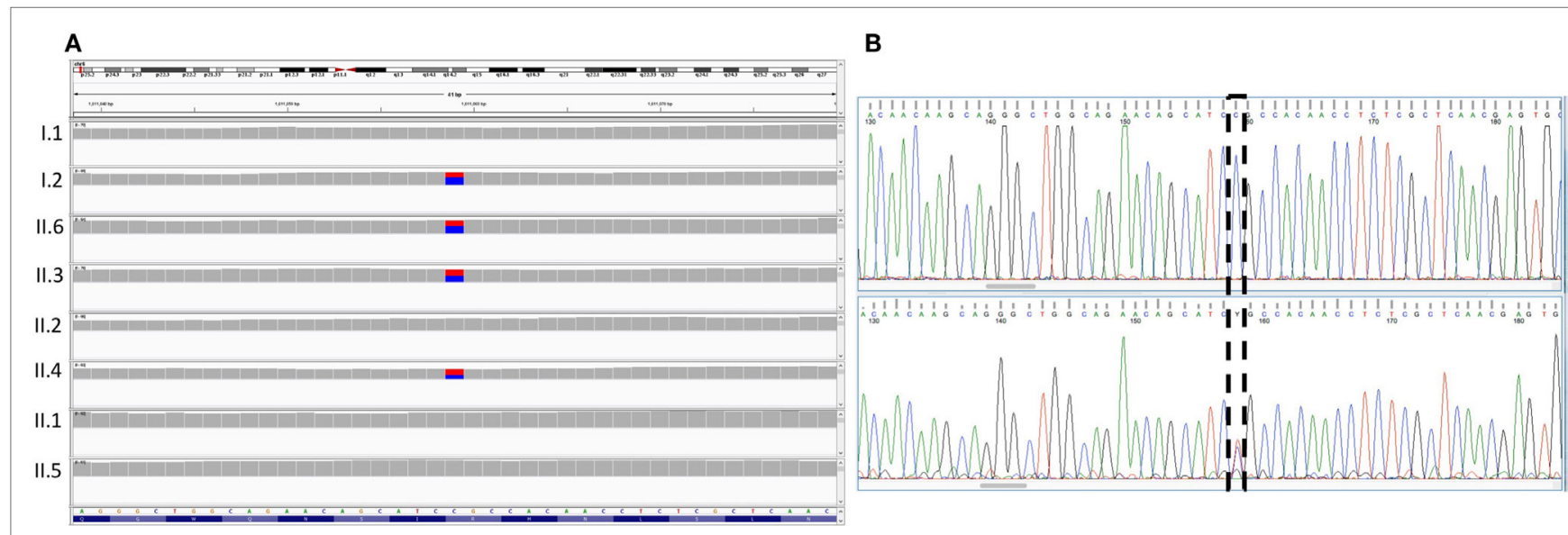

FIGURE 2 | Sequencing results for the FOXC1 variant p.R127C. (A) Integrative genomics viewer visualization of whole exome sequencing shows a novel heterozygous variant (blue for the normal "C" allele and red for the variant "T" allele) in FOXC1 gene in family members I.2, II.3, II.2, and II.6. (B) Sanger sequencing of the FOXC1 gene confirmed the C>T variant (boxed), in the affected individuals represented in the lower panel versus the normal individuals in the upper panel. 
TABLE 2 | Variants shared by affected patients inherited exclusively from the father.

\begin{tabular}{|c|c|c|c|c|c|c|c|c|c|c|c|c|c|c|c|c|c|c|c|c|}
\hline Sample & Gene & Variant & Coordinate & Chr & Type & Filters & Quality & $\begin{array}{l}\text { Inherited } \\
\text { from }\end{array}$ & $\begin{array}{l}\text { Allelic } \\
\text { depths }\end{array}$ & Transcript & Consequence & $\begin{array}{l}\text { Protein } \\
\text { position }\end{array}$ & $\begin{array}{l}\text { Amino } \\
\text { acids }\end{array}$ & Sift & PolyPhen & dbSNP ID & $\begin{array}{l}\text { Allele freq } \\
\text { global minor }\end{array}$ & $\begin{array}{l}\text { Allele freq } \\
\text { EVS }\end{array}$ & $\begin{array}{c}\text { EVS } \\
\text { coverage }\end{array}$ & $\begin{array}{c}\text { EvS } \\
\text { samples }\end{array}$ \\
\hline 11.3 & DPT & $T>T / C$ & 16870348 & 1 & Snv & PASS & 805.77 & Father & 34,28 & NM_001937.4 & missense_variant & 149 & Y/C & deleterious(0.04) & $\begin{array}{l}\text { possibly_ } \\
\text { damaging(0.628) }\end{array}$ & & 0 & 0 & 172 & 6,503 \\
\hline 11.4 & DPT & $T>T / C$ & 16870348 & 1 & Snv & PASS & 997.77 & Father & 23,30 & NM_001937.4 & missense_variant & 149 & Y/C & deleterious(0.04) & $\begin{array}{l}\text { possibly_ } \\
\text { damaging(0.628) }\end{array}$ & & 0 & 0 & 172 & 6,503 \\
\hline 11.6 & DPT & $T>T / C$ & 16870348 & 1 & Snv & PASS & 548.77 & Father & 28,21 & NM_001937.4 & missense_variant & 149 & Y/C & deleterious(0.04) & $\begin{array}{l}\text { possibly_ } \\
\text { damaging(0.628) }\end{array}$ & & 0 & 0 & 172 & 6,503 \\
\hline 11.3 & INHBA & $\mathrm{C}>\mathrm{C} / \mathrm{T}$ & 41729843 & 7 & Snv & PASS & 798.77 & Father & 28,27 & NR_027118.1 & upstream_gene_variant & 0 & & & & rs138819536 & 0.09 & 0.26 & 51 & 6,503 \\
\hline 11.4 & INHBA & $\mathrm{C}>\mathrm{C} / \mathrm{T}$ & 41729843 & 7 & Snv & PASS & 988.77 & Father & 29,33 & NR_027118.1 & upstream_gene_variant & 0 & & & & rs138819536 & 0.09 & 0.26 & 51 & 6,503 \\
\hline 11.6 & INHBA & $\mathrm{C}>\mathrm{C} / \mathrm{T}$ & 41729843 & 7 & Snv & PASS & 774.77 & Father & 21,27 & NR_027118.1 & upstream_gene_variant & 0 & & & & rs138819536 & 0.09 & 0.26 & 51 & 6,503 \\
\hline 11.3 & $\begin{array}{l}\text { POLR2J4, } \\
\text { SPDYE1 }\end{array}$ & $G>G / A$ & 44046965 & 7 & Snv & PASS & $1,115.77$ & Father & 63,42 & NR_003655.2 & intron_variant, nc_transcript_variant & 0 & & & & rs141407881 & 0.55 & 1.05 & 174 & 6,503 \\
\hline 11.4 & $\begin{array}{l}\text { POLR2J4, } \\
\text { SPDYE1 }\end{array}$ & $G>G / A$ & 44046965 & 7 & Snv & PASS & 978.77 & Father & 54,37 & NR_003655.2 & intron_variant, nc_transcript_variant & 0 & & & & rs141407881 & 0.55 & 1.05 & 174 & 6,503 \\
\hline 11.6 & $\begin{array}{l}\text { POLR2J4, } \\
\text { SPDYE1 }\end{array}$ & $G>G / A$ & 44046965 & 7 & Snv & PASS & 966.77 & Father & 44,38 & NR_003655.2 & intron_variant, nc_transcript_variant & 0 & & & & rs141407881 & 0.55 & 1.05 & 174 & 6,503 \\
\hline 11.3 & RBM43 & $C>C / G$ & 152112161 & 2 & Snv & PASS & 907.77 & Father & 21,37 & NM_198557.2 & missense_variant & 34 & V/L & tolerated(0.8) & benign(0) & rs147060862 & 1.56 & 2.39 & 130 & 6,503 \\
\hline 11.4 & RBM43 & $\mathrm{C}>\mathrm{C} / \mathrm{G}$ & 152112161 & 2 & Snv & PASS & 680.77 & Father & 22,28 & NM_198557.2 & missense_variant & 34 & $V / L$ & tolerated(0.8) & benign(0) & rs147060862 & 1.56 & 2.39 & 130 & 6,503 \\
\hline 11.6 & RBM43 & $C>C / G$ & 152112161 & 2 & Snv & PASS & 278.77 & Father & 26,12 & NM_198557.2 & missense_variant & 34 & $V / L$ & tolerated(0.8) & benign(0) & rs147060862 & 1.56 & 2.39 & 130 & 6,503 \\
\hline 11.3 & $\begin{array}{l}\text { RTEL1, } \\
\text { RTEL1-TNFRSF6B }\end{array}$ & $G>G / A$ & 62324290 & 20 & Snv & PASS & $1,215.77$ & Father & 46,47 & NM_003823.3 & upstream_gene_variant & 0 & & & & rs61736615 & 1.69 & 2.81 & 118 & 6,492 \\
\hline 11.4 & $\begin{array}{l}\text { RTEL1, } \\
\text { RTEL1-TNFRSF6B }\end{array}$ & $G>G / A$ & 62324290 & 20 & Snv & PASS & 941.77 & Father & 44,35 & NM_003823.3 & upstream_gene_variant & 0 & & & & rs61736615 & 1.69 & 2.81 & 118 & 6,492 \\
\hline 11.6 & $\begin{array}{l}\text { RTEL1, } \\
\text { RTEL1-TNFRSF6B }\end{array}$ & $G>G / A$ & 62324290 & 20 & Snv & PASS & $1,239.77$ & Father & 34,47 & NM_003823.3 & upstream_gene_variant & 0 & & & & rs61736615 & 1.69 & 2.81 & 118 & 6,492 \\
\hline 11.3 & TIPRL & $\mathrm{C}>\mathrm{C} / \mathrm{T}$ & $1.68 \mathrm{E}+08$ & 1 & Snv & PASS & $1,016.77$ & Father & 36,32 & NM_152902.3 & splice_region_variant, intron_variant & 0 & & & & & 0 & 0.01 & 107 & 6,503 \\
\hline 11.4 & TIPRL & $\mathrm{C}>\mathrm{C} / \mathrm{T}$ & $1.68 \mathrm{E}+08$ & 1 & Snv & PASS & 573.77 & Father & 41,20 & NM_152902.3 & splice_region_variant, intron_variant & 0 & & & & & 0 & 0.01 & 107 & 6,503 \\
\hline 11.6 & TIPRL & $\mathrm{C}>\mathrm{C} / \mathrm{T}$ & $1.68 \mathrm{E}+08$ & 1 & Snv & PASS & 893.77 & Father & 30,30 & NM_152902.3 & splice_region_variant, intron_variant & 0 & & & & & 0 & 0.01 & 107 & 6,503 \\
\hline
\end{tabular}

EVS, Exome databases. 
TABLE 3 | Variants shared by patients with both glaucoma and congenital heart disease inherited either from the father alone or from both parents.

\begin{tabular}{|c|c|c|c|c|c|c|c|c|c|c|c|c|c|c|c|c|c|c|c|c|}
\hline Sample & Gene & Variant & Coordinate & $\mathrm{Chr}$ & Type & Filters & Quality & $\begin{array}{l}\text { Inherited } \\
\text { from }\end{array}$ & $\begin{array}{l}\text { Allelic } \\
\text { depths }\end{array}$ & Transcript & Consequence & $\begin{array}{l}\text { Protein } \\
\text { position }\end{array}$ & $\begin{array}{l}\text { Amino } \\
\text { acids }\end{array}$ & Sift & PolyPhen & dbSNP ID & $\begin{array}{l}\text { Allele freq } \\
\text { global } \\
\text { minor }\end{array}$ & $\begin{array}{l}\text { Allele } \\
\text { freq } \\
\text { EVS }\end{array}$ & $\begin{array}{l}\text { EvS } \\
\text { coverage }\end{array}$ & $\begin{array}{c}\text { EVs } \\
\text { samples }\end{array}$ \\
\hline 11.3 & CCDC155 & $\mathrm{C}>\mathrm{C} / \mathrm{G}$ & 49910139 & 19 & Snv & PASS & 816.77 & Father & 49,31 & NM_144688.4 & $\begin{array}{l}\text { splice_region_variant, } \\
\text { intron_variant }\end{array}$ & 0 & & & & rs 112074780 & 1.1 & 1.1 & 47 & 6,098 \\
\hline 11.6 & CCDC155 & $\mathrm{C}>\mathrm{C} / \mathrm{G}$ & 49910139 & 19 & Snv & PASS & $1,090.77$ & Father & 54,39 & NM_144688.4 & $\begin{array}{l}\text { splice_region_variant, } \\
\text { intro_variant }\end{array}$ & 0 & & & & rs112074780 & 1.1 & 1.1 & 47 & 6,098 \\
\hline 11.3 & CCDC9 & $T>T / C$ & 47768055 & 19 & Snv & PASS & 529.77 & Father & 41,22 & NM_015603.2 & missense_variant & 191 & V/A & deleterious $(0.01)$ & unknown(0) & & 0 & 0 & 19 & 6,496 \\
\hline 11.6 & $\operatorname{CCDC9}$ & $T>T / C$ & 47768055 & 19 & Snv & PASS & 630.77 & Father & 40,28 & NM_015603.2 & missense_variant & 191 & V/A & deleterious(0.01) & unknown(0) & & 0 & 0 & 19 & 6,496 \\
\hline 11.3 & DKKL1 & $\mathrm{G}>\mathrm{G} / \mathrm{A}$ & 49878275 & 19 & Snv & PASS & $1,317.77$ & Father & 31,45 & NM_014419.3 & missense_variant & 240 & $R / Q$ & tolerated(1) & benign(0.002) & rs353899403 & 0.73 & 0.54 & 25 & 6,444 \\
\hline 11.6 & DKKL1 & $G>G / A$ & 49878275 & 19 & Snv & PASS & 882.77 & Father & 40,32 & NM_014419.3 & missense_variant & 240 & $R / Q$ & tolerated(1) & benign(0.002) & rs35389403 & 0.73 & 0.54 & 25 & 6,444 \\
\hline 11.3 & KLK11 & $\mathrm{C}>\mathrm{C} / \mathrm{T}$ & 51527970 & 19 & Snv & PASS & 781.77 & Father & 51,34 & NM_144947.1 & missense_variant & 73 & $E / K$ & tolerated(0.31) & benign(0.178) & rs117268623 & 1.74 & 1.94 & 26 & 6,503 \\
\hline 11.6 & KLK11 & $\mathrm{C}>\mathrm{C} / \mathrm{T}$ & 51527970 & 19 & Snv & PASS & 772.77 & Father & 34,31 & NM_144947.1 & missense_variant & 73 & $\mathrm{E} / \mathrm{K}$ & tolerated(0.31) & benign(0.178) & rs117268623 & 1.74 & 1.94 & 26 & 6,503 \\
\hline 11.3 & LAMC2 & $\mathrm{G}>\mathrm{G} / \mathrm{A}$ & 183177132 & 1 & Snv & PASS & 808.77 & Father & 25,28 & NM_005562.2 & missense_variant & 66 & $E / K$ & deleterious(0) & $\begin{array}{l}\text { probably_ } \\
\text { damaging(0.995) }\end{array}$ & rs146325169 & 0 & 0.12 & 275 & 6,503 \\
\hline 11.6 & LAMC2 & $\mathrm{G}>\mathrm{G} / \mathrm{A}$ & 183177132 & 1 & Snv & PASS & 627.77 & Father & 21,27 & NM_005562.2 & missense_variant & 66 & $E / K$ & deleterious(0) & $\begin{array}{l}\text { probably_ } \\
\text { damaging(0.995) }\end{array}$ & rs146325169 & 0 & 0.12 & 275 & 6,503 \\
\hline 11.3 & OR8U1 & $\mathrm{C}>\mathrm{C} / \mathrm{A}$ & 56143976 & 11 & Snv & PASS & 61.77 & Father & 25,4 & NM_001005204.1 & missense_variant & 293 & $\mathrm{Q} / \mathrm{K}$ & deleterious $(0.03)$ & benign(0.003) & & 0 & 0 & 123 & 6,047 \\
\hline 11.6 & OR8U1 & $\mathrm{C}>\mathrm{C} / \mathrm{A}$ & 56143976 & 11 & Snv & PASS & 43.77 & Father & 17,3 & NM_001005204.1 & missense_variant & 293 & $\mathrm{Q} / \mathrm{K}$ & deleterious(0.03) & benign(0.003) & & 0 & 0 & 123 & 6,047 \\
\hline 11.3 & PDILT & $A>A / C$ & 20371972 & 16 & Snv & PASS & 511.77 & Father & 14,18 & NM_174924.1 & missense_variant & 475 & LR & tolerated(0.31) & benign(0.002) & rs 4500734 & 1.37 & 2.41 & 138 & 6,503 \\
\hline 11.6 & PDILT & $A>A / C$ & 20371972 & 16 & Snv & PASS & 283.77 & Father & 17,12 & NM_174924.1 & missense_variant & 475 & L/R & tolerated(0.31) & benign(0.002) & rs4500734 & 1.37 & 2.41 & 138 & 6,503 \\
\hline 11.3 & PIH1D1 & $\mathrm{G}>\mathrm{G} / \mathrm{A}$ & 49949912 & 19 & Snv & PASS & 354.77 & Father & 15,14 & NM_017916.2 & missense_variant & 243 & $\mathrm{R} / \mathrm{C}$ & tolerated(0.07) & $\begin{array}{l}\text { possibly_ } \\
\text { damaging(0.866) }\end{array}$ & rs149419497 & 0.41 & 0.34 & 61 & 6,503 \\
\hline 11.6 & PIH1D1 & $\mathrm{G}>\mathrm{G} / \mathrm{A}$ & 49949912 & 19 & Snv & PASS & 304.77 & Father & 19,14 & NM_017916.2 & missense_variant & 243 & $\mathrm{R} / \mathrm{C}$ & tolerated(0.07) & $\begin{array}{l}\text { possibly_ } \\
\text { damaging(0.866) }\end{array}$ & rs149419497 & 0.41 & 0.34 & 61 & 6,503 \\
\hline 11.3 & PRX & $\mathrm{G}>\mathrm{G} / \mathrm{A}$ & 40900763 & 19 & Snv & PASS & $1,056.77$ & Father & 37,36 & NM_181882.2 & missense_variant & 1166 & P/S & tolerated(0.55) & benign(0.002) & rs147826200 & 0 & 0.07 & 72 & 6,503 \\
\hline 11.6 & PRX & $G>G / A$ & 40900763 & 19 & Snv & PASS & $1,039.77$ & Father & 30,39 & NM_181882.2 & missense_variant & 1166 & $\mathrm{P} / \mathrm{S}$ & tolerated(0.55) & benign(0.002) & rs 147826200 & 0 & 0.07 & 72 & 6,503 \\
\hline 11.3 & RYR1 & $\mathrm{G}>\mathrm{G} / \mathrm{A}$ & 38997024 & 19 & Snv & PASS & 717.77 & Father & 26,25 & NM_000540.2 & $\begin{array}{l}\text { splice_region_variant, } \\
\text { intron_variant }\end{array}$ & 0 & & & & rs200023171 & 0.05 & 0.03 & 50 & 6,503 \\
\hline 11.6 & RYR1 & $G>G / A$ & 38997024 & 19 & Snv & PASS & 529.77 & Father & 20,18 & NM_000540.2 & $\begin{array}{l}\text { splice_region_variant, } \\
\text { intro_variant }\end{array}$ & 0 & & & & rs200023171 & 0.05 & 0.03 & 50 & 6,503 \\
\hline 11.3 & SHCBP1L & $A>A / G$ & 182909488 & 1 & Snv & PASS & 928.77 & Father & 15,30 & NM_030933.2 & missense_variant & 249 & $1 / T$ & tolerated(0.74) & $\begin{array}{l}\text { probably_ } \\
\text { damaging(0.996) }\end{array}$ & rs116513797 & 1.28 & 0.43 & 114 & 6,503 \\
\hline 11.6 & SHCBP1L & $A>A / G$ & 182909488 & 1 & Snv & PASS & 798.77 & Father & 15,26 & NM_030933.2 & missense_variant & 249 & $1 / T$ & tolerated(0.74) & $\begin{array}{l}\text { probably_ } \\
\text { damaging(0.996) }\end{array}$ & rs116513797 & 1.28 & 0.43 & 114 & 6,503 \\
\hline 11.3 & SIX5 & $\mathrm{C}>\mathrm{C} / \mathrm{A}$ & 46269196 & 19 & Snv & PASS & $1,634.77$ & Father & 57,59 & NM_175875.4 & missense_variant & 595 & $\mathrm{~V} / \mathrm{L}$ & tolerated(0.16) & $\begin{array}{l}\text { possibly_- } \\
\text { damaging(0.658) }\end{array}$ & rs114060947 & 2.24 & 0 & 14 & 6,478 \\
\hline 11.6 & SIX5 & $\mathrm{C}>\mathrm{C} / \mathrm{A}$ & 46269196 & 19 & Snv & PASS & 897.77 & Father & 62,35 & NM_175875.4 & missense_variant & 595 & V/L & tolerated(0.16) & $\begin{array}{l}\text { possibly_ } \\
\text { damaging(0.658) }\end{array}$ & rs114060947 & 2.24 & 0 & 14 & 6,478 \\
\hline 11.3 & STK10 & $G>G / A$ & 171481667 & 5 & Snv & PASS & 585.77 & Father & 33,25 & NM_005990.3 & missense_variant & 853 & $S / L$ & tolerated(0.07) & benign(0.146) & rs56066852 & 0.55 & 0.99 & 132 & 6,503 \\
\hline 11.6 & STK10 & $\mathrm{G}>\mathrm{G} / \mathrm{A}$ & 171481667 & 5 & Snv & PASS & 419.77 & Father & 32,19 & NM_005990.3 & missense_variant & 853 & $S / L$ & tolerated(0.07) & benign(0.146) & rs56066852 & 0.55 & 0.99 & 132 & 6,503 \\
\hline 11.3 & ZNF229 & $\mathrm{G}>\mathrm{G} / \mathrm{A}$ & 44934187 & 19 & Snv & PASS & 974.77 & Father & 53,39 & NM_014518.2 & missense_variant & 257 & $R / C$ & deleterious(0.01) & benign(0.059) & rs 144097942 & 0.55 & 1.24 & 106 & 6,015 \\
\hline 11.6 & ZNF229 & $\mathrm{G}>\mathrm{G} / \mathrm{A}$ & 44934187 & 19 & Snv & PASS & 534.77 & Father & 41,23 & NM_014518.2 & missense_variant & 257 & $\mathrm{R} / \mathrm{C}$ & deleterious $(0.01)$ & benign(0.059) & rs144097942 & 0.55 & 1.24 & 106 & 6,015 \\
\hline
\end{tabular}


TABLE 3 | Continued

Sample Gene Variant Coordinate Chr Type Filters Ouality Inherited Allelic Transcript Consequence Protein Amino Sift

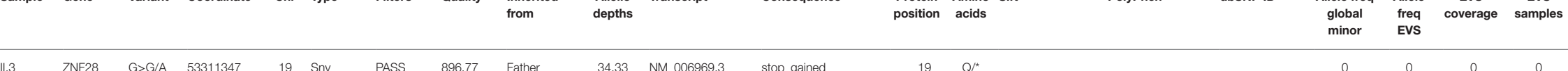

\begin{tabular}{|c|c|c|c|c|c|c|c|c|c|c|c|c|c|c|c|c|c|c|c|c|}
\hline 11.3 & ZNF28 & $G>G / A$ & 53311347 & 19 & Snv & PASS & 896.77 & Father & 34,33 & NM_006969.3 & stop_gained & 19 & $Q^{/ *}$ & & & & 0 & 0 & 0 & 0 \\
\hline 11.6 & ZNF28 & $G>G / A$ & 53311347 & 19 & Snv & PASS & 410.77 & Father & 22,18 & NM_006969.3 & stop_gained & 19 & $Q^{/ *}$ & & & & 0 & 0 & 0 & 0 \\
\hline 11.3 & ZNF43 & $T>T / T C$ & 21992330 & 19 & Insertion & PASS & 125.77 & Father & 11,6 & NM_001256653.1 & $\begin{array}{l}\text { frameshift_variant, } \\
\text { feature_elongation }\end{array}$ & 179 & & & & & 0 & 0 & 0 & 0 \\
\hline 11.6 & ZNF43 & $T>T / T C$ & 21992330 & 19 & Insertion & PASS & 185.77 & Father & 9,7 & NM_001256653.1 & $\begin{array}{l}\text { frameshift_variant, } \\
\text { feature_elongation }\end{array}$ & 179 & & & & & 0 & 0 & 0 & 0 \\
\hline 11.3 & ZNF616 & $T>T / C$ & 52618555 & 19 & Snv & PASS & 581.77 & Father & 26,20 & NM_178523.3 & missense_variant & 621 & $\mathrm{~N} / \mathrm{S}$ & tolerated(0.3) & benign $(0.007)$ & rs116130534 & 0.05 & 0 & 69 & 6,503 \\
\hline 11.6 & ZNF616 & $T>T / C$ & 52618555 & 19 & Snv & PASS & 775.77 & Father & 18,24 & NM_178523.3 & missense_variant & 621 & N/S & tolerated(0.3) & benign $(0.007)$ & rs116130534 & 0.05 & 0 & 69 & 6,503 \\
\hline 11.3 & ZNF765 & $T>T / G$ & 53912045 & 19 & Snv & PASS & $1,518.77$ & Father & 103,59 & NM_001040185.1 & missense_variant & 413 & $\mathrm{C} / \mathrm{G}$ & deleterious $(0)$ & $\begin{array}{l}\text { probably__ } \\
\text { damaging(0.997) }\end{array}$ & & 0 & 0.02 & 96 & 6,503 \\
\hline
\end{tabular}

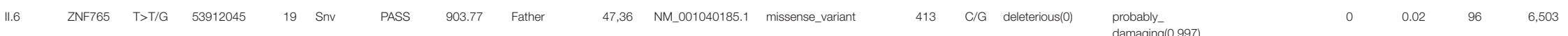

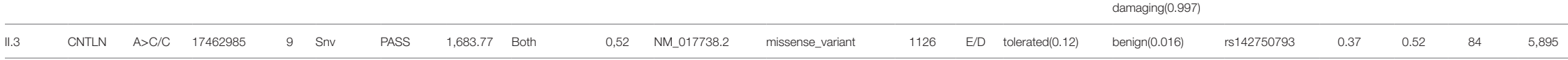

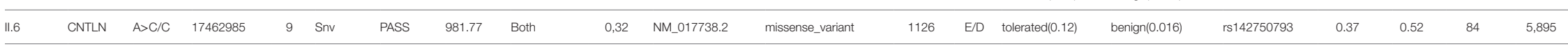

11.3 DPP4 $\quad$ G>C/C $\quad 162903930 \quad 2 \quad$ Snv $\quad$ PASS $\quad 1,135.77 \quad$ B

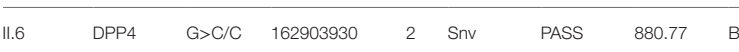

$\begin{array}{llllllll}\text { II.3 } & \text { KIR2DL1 } & \text { A>G/G } & 55285072 & 19 & \text { Snv } & \text { PASS } & 1,063.77\end{array}$

$\begin{array}{llllllllll}11.6 & \text { KIR2DL1 } & A>G / G & 55285072 & 19 & \text { Snv } & \text { PASS } & 840.77 \quad \text { B } & & \end{array}$

$\begin{array}{llllllll}11.3 & \text { LLLRB5 } & \text { G>ANA } & 54756415 & 19 & \text { Snv } & \text { PASS } & 890.77\end{array}$

11.6 LILRB5 $\quad$ G>ANA $54756415 \quad 19 \quad$ Snv $\quad$ PASS $\quad 650.77 \quad$ B

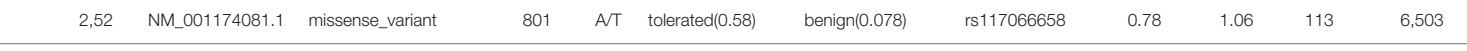

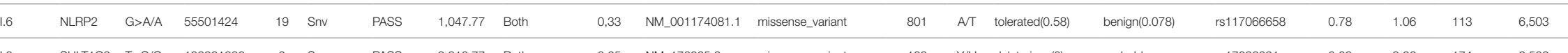

11.6 SULT1C2 2 T>C/C $108921036 \quad 2$ Snv $\quad$ PASS $\quad 1,496.77$ Both $\quad 0,45 \quad$ NM_176825.2 missense_varian

probably

ramaging(1)

\begin{tabular}{lllllllllllllllllllll}
\hline 11.3 & TCP10L2 & T>C/C & 167592524 & 6 & Snv & PASS & $1,270.77$ & Both & 0,37 & NM_001145121.1 & missense_variant & 228 & LP & tolerated(0.13) & benign(0) & rs2989545 & 0 & 0 & 24 & 2,278
\end{tabular}

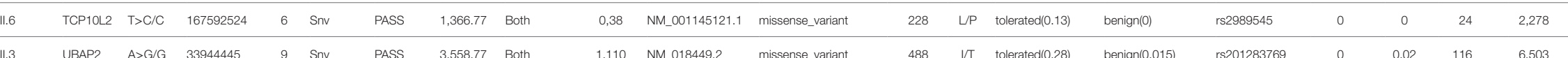

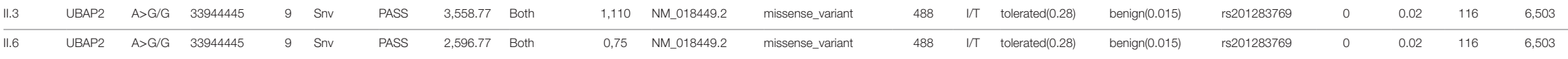

EVS, Exome databases. 
TABLE 4 | Variants inherited from the father alone and not present in other siblings.

\section{Sample Gene varint \\ Filters Quality Inherited Allelic}

Transcript

Consequence

Protein Amino Sif

PolyPhen

dbSNP ID

Allele Allele EvS EvS

Freq Freq Coverage Samples

Global EVs

\begin{tabular}{|c|c|c|c|c|c|c|c|c|c|c|c|c|c|c|c|c|c|c|c|c|}
\hline 11.3 & GXYLT1 & $\mathrm{C}>\mathrm{C} / \mathrm{A}$ & 42538340 & 12 & Snv & PASS & 130.77 & Father & 3,3 & NM_173601.1 & missense_variant & 37 & $\mathrm{G} / \mathrm{C}$ & tolerated(0.1) & benign(0.186) & & 0 & 0 & 3 & 4104 \\
\hline II.3 & GXYLT1 & $T>T / C$ & 42538349 & 12 & Snv & PASS & 46.77 & Father & 3,2 & NM_173601.1 & missense_variant & 34 & $T / A$ & tolerated(0.78) & benign(0) & & 0 & 0 & 4 & 4818 \\
\hline 11.3 & GXYLT1 & $\mathrm{C}>\mathrm{C} / \mathrm{A}$ & 42538352 & 12 & Snv & PASS & 46.77 & Father & 3,2 & NM_173601.1 & stop_gained & 33 & $\mathrm{G} /{ }^{*}$ & & & & 0 & 0 & 4 & 4931 \\
\hline 11.3 & GXYLT1 & $A>A / T$ & 42538366 & 12 & Snv & PASS & 46.77 & Father & 3,2 & NM_173601.1 & missense_variant & 28 & V/E & tolerated(0.27) & benign(0.045) & & 0 & 0 & 5 & 5228 \\
\hline 11.3 & GXYLT1 & $\mathrm{C}>\mathrm{C} / \mathrm{T}$ & 42538367 & 12 & Snv & PASS & 46.77 & Father & 3,2 & NM_173601.1 & missense_variant & 28 & $\mathrm{~V} / \mathrm{M}$ & tolerated(0.22) & benign(0.161) & & 0 & 0 & 5 & 5232 \\
\hline 11.3 & MTFMT & $C A>C A / C$ & 65312614 & 15 & Deletion & PASS & 35.77 & Father & 2,3 & NM_139242.3 & $\begin{array}{l}\text { splice_region_variant, intron_ } \\
\text { variant, feature_truncation }\end{array}$ & 0 & & & & & 0 & 0 & 17 & 5906 \\
\hline 11.3 & MUC16 & $A>A / A G$ & 9012894 & 19 & Insertion & PASS & 913.77 & Father & 95,30 & NM_024690.2 & $\begin{array}{l}\text { frameshift_variant, } \\
\text { feature_elongation }\end{array}$ & 12,850 & & & & & 0 & 0 & 0 & 0 \\
\hline II.3 & MUC16 & $A G>A G / A$ & 9012897 & 19 & Deletion & PASS & 931.77 & Father & 96,28 & NM_024690.2 & $\begin{array}{l}\text { frameshift_variant, splice_region_ } \\
\text { variant, feature_truncation }\end{array}$ & 12,849 & & & & & 0 & 0 & 211 & 6170 \\
\hline 11.3 & PAXBP1 & $\mathrm{C}>\mathrm{C} / \mathrm{T}$ & 34133365 & 21 & Snv & PASS & 322.77 & Father & 12,13 & NM_016631.3 & $\begin{array}{l}\text { splice_region_variant, } \\
\text { intron_variant }\end{array}$ & 0 & & & & rs111951332 & 1.01 & 1.71 & 179 & 6503 \\
\hline 11.3 & SDHAP1 & $A>A / G$ & 195690163 & 3 & Snv & PASS & 659.77 & Father & 82,28 & NR_003264.2 & $\begin{array}{l}\text { splice_region_variant, intron_ } \\
\text { variant, nc_transcript_variant }\end{array}$ & 0 & & & & rs201372496 & 0 & 0 & 0 & 0 \\
\hline
\end{tabular}

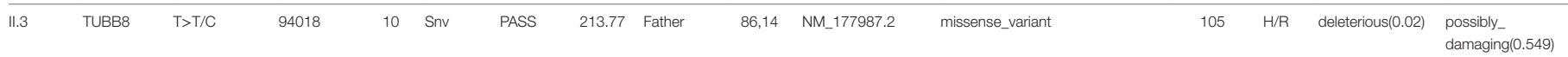

Splice region_variant, intron

variant, feature_elongation

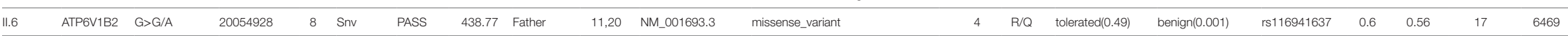

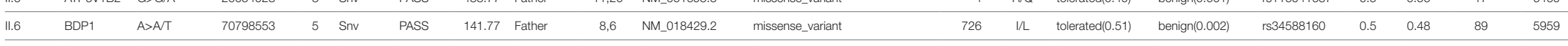

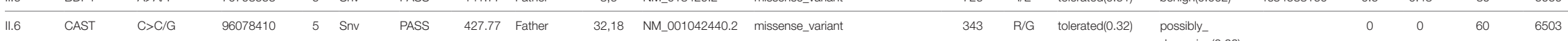

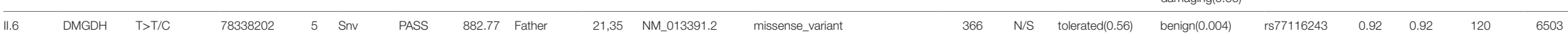

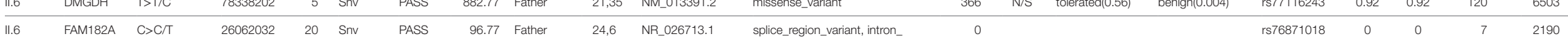

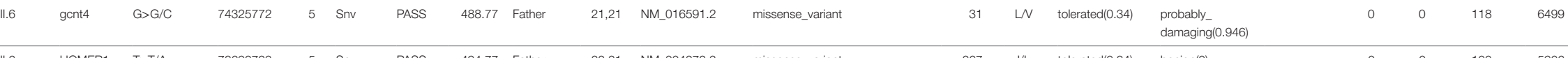

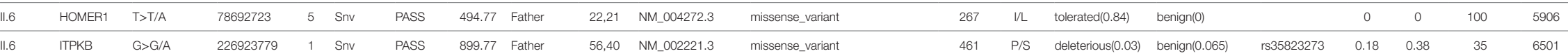

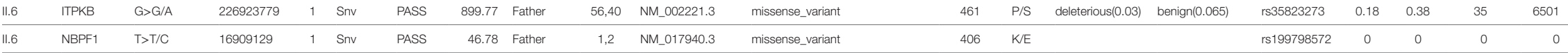

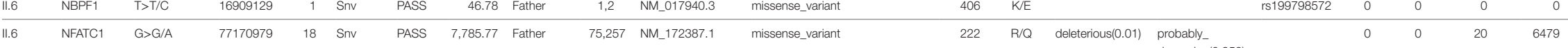

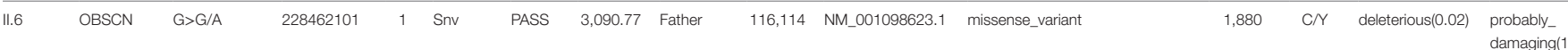

damaging(0.953)

I1.6 $\quad$ PCDHA1, G>G/C $140222138 \quad 5 \quad$ Snv $\quad$ PASS $1,215.77$ Father $84,37 \quad$ NM_018910.2 intron varian

PCDHA2,

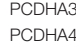

PCDHA5

PCDHAG,

PCDHA7,

PCDHA8 


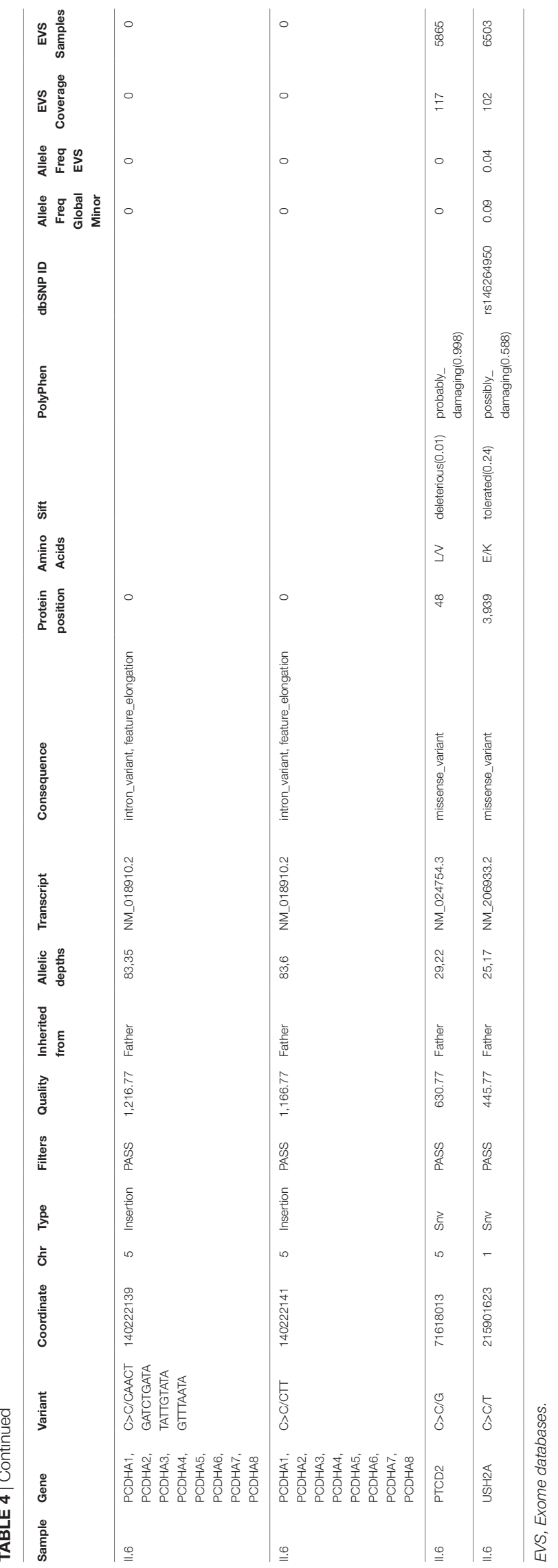

linked to severe functional alterations of either FOXC1 and/ or PITX2 (28-30). The mutations associated with the ARS phenotype can range from frameshift mutations resulting in premature termination of translation in the forkhead domain or homeodomain, missense mutations reducing transactivation and protein interactions, and nonsense mutations causing haploinsufficiency of the gene product. Most of these mutations will hamper the stability of either one of these transcription factors protein complex over the DNA, and thus have a deleterious effect on transcriptional regulation of target genes $(26,31)$. Most of the mutations in FOXC1 appear to be linked to isolated ocular defects or ocular, combined with cardiac, skeletal, and auditory defects. In this study, two of the children who suffered from glaucoma also suffered from CHD. Their mother however, had the same FOXC1 variant, but did not present with glaucoma or $\mathrm{CHD}$, though she could have defects pertaining to anterior segment dysgenesis. This clinical heterogeneity suggested an important role for modifier factors (genetic, environmental, and/or stochastic) on the phenotypic outcomes. We are thus proposing a digenic model to account for some of the phenotypes in this particular family as deduced from a thorough analysis using WES.

\section{Ocular Phenotype: Does FOXC1 Need DPT for Glaucoma?}

Our results do point out to the determinant role of the p.R127C variant in the ocular phenotype manifested in the three affected children, and in particular to glaucoma which was manifested at very early ages. Only those individuals with this variant do have glaucoma with the exception of the mother, who is a carrier but is glaucoma-free. In search for modifier genes using WES, we could not detect any variation in genes pertaining to the anterior segment dysgenesis phenotype in general and to glaucoma in particular (Figure $\mathbf{1}$ and Table 1). In parallel, our analysis of the missense variant shows that it is novel and never reported before in the literature. However, the arginine amino acid at position 127 was linked to prior cases of ARS. In particular, a p.R127H variant was associated with an ARS case with the affected proband having severe ocular defects and glaucoma (32). As in our case, this variant was inherited from his mother who does not have glaucoma, but has typical posterior embryotoxon. More recently, a case of ARS was linked to a missense mutation at the same position leading: the p.R127L variant was found in a proband with characteristics of ARS including glaucoma and a cardiac structural defect due to a PDA (33). The patient inherited this variant from his father who was only diagnosed with glaucoma, while the patient's sister was not available for genetic testing since she passed away as a result of severe dilated cardiomyopathy. As in the published two cases, and in other cases whereby the severity of the ARS phenotype is more pronounced in the children versus their parents $(32,34)$, we hypothesize that a modifier variant inherited from the FOXC1 "variant-free parent" would account for the severity of the phenotype in the children versus their parents. Our WES results identified a novel variant in the $D P T$ gene inherited from the father and only present in the affected children with glaucoma but not in the healthy children. It could explain the 


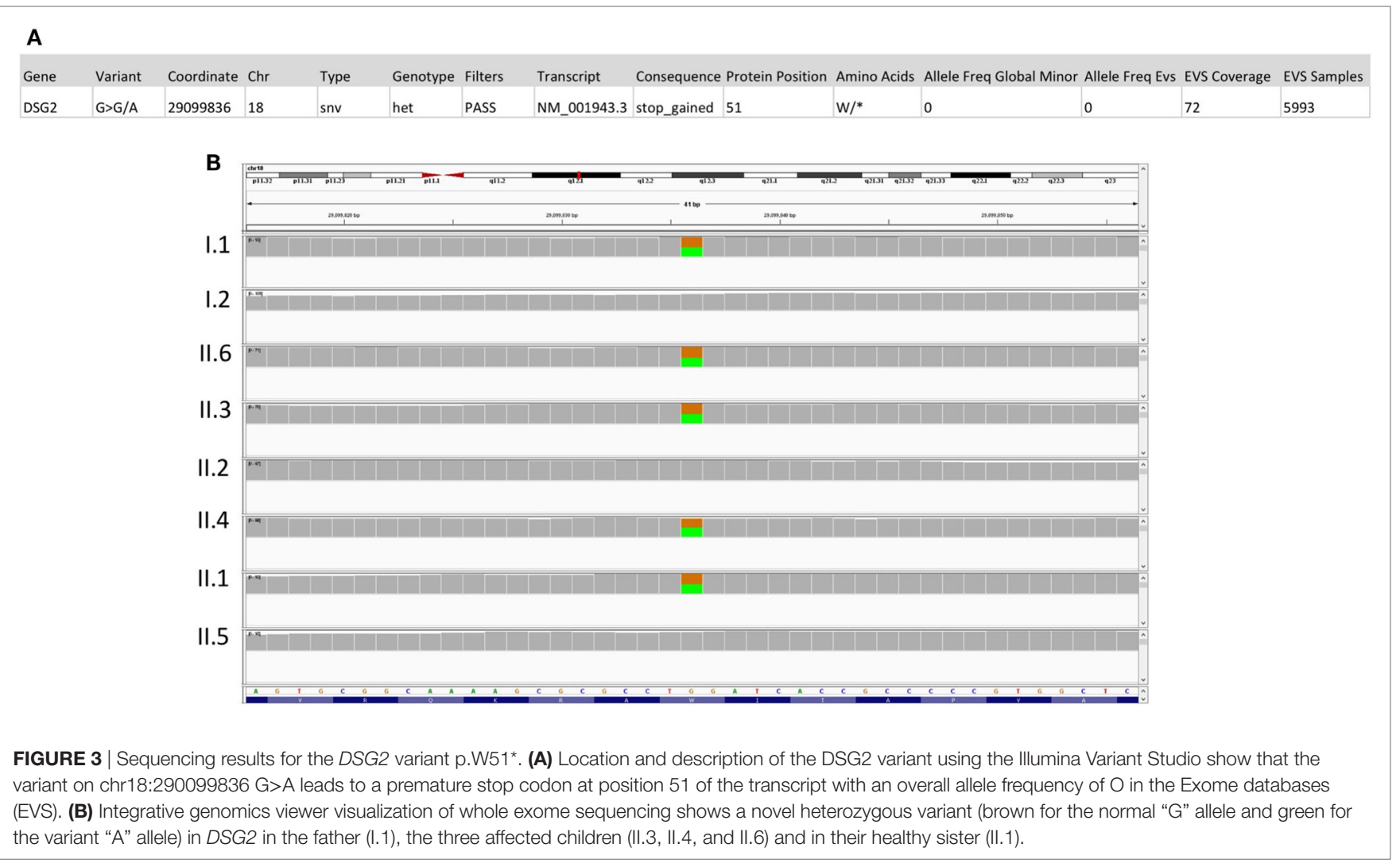

early onset of the anterior segment dysgenesis phenotype and particularly glaucoma in this case. The p.Y149C missense variant is only found in the three affected children with glaucoma: it is predicted to be deleterious and is neither found in healthy Lebanese controls, nor in the gnomAD database (www.gnomad. broadinstitute.org). Additionally, there were no previous reports on the role of DPT in glaucoma, and only a few publications describe its expression and role in ocular development and pathology. DPT is an extracellular matrix protein required for the organization of collagen in the skin, as depicted in the mouse knockout model (35). This latter did show also a defect in the corneal matrix organization, which coupled with the prominent expression of the protein in the optic nerve in zebrafish suggest a potential role in ocular development $(36,37)$. We do suggest a genetic/molecular interaction between DPT and FOXC1, which would be largely disrupted by the missense mutations detected in our case. A double-heterozygous mouse model carrying only one copy of each gene would potentially yield better insight into this proposed interaction.

\section{Cardiac Phenotype: A FOXC1/NFATC1 Genetic Interaction}

The cardiac phenotype in the indexed-family is divided into two: a mild VSD not requiring any intervention and a severe TOFlike phenotype that required major intervention (Figure 1). We sought that differential variants inherited from the father would contribute to this differential expressivity of the ARS phenotype within the three affected children in this family: two with a cardiac phenotype and the third with only glaucoma. Interestingly, we unravel two novel missense mutations in OBSCN (p.C1880Y) and NFATC1 (p.R222Q) that are predicted to be damaging (Table 4). Both variants are neither present in the Lebanese controls nor in the genome and exome databases. Given that $O B C S N$ mutations have not been linked to $\operatorname{CHD}(38,39)$, but could be potentially causing cardiomyopathies, we hypothesize that the NFATC1 missense variant along the FOXC1 variant is responsible for the cardiac phenotype. Our rationale is based on previous findings from our group that only a compound mutation in NFATC1 could be linked to a cardiac phenotype (40), which is also mirrored in the knockout mouse model for $\mathrm{Nfatc1}$ that shows that the absence of both $N f a t c 1$ alleles is required to have a severe cardiac phenotype while the heterozygous mice are healthy $(41,42)$. Both FOXC1 and NFATC1 are expressed in the secondary heart field, and thus could be implicated in common transcriptional pathways that shape up the cardiac valves, and septation of the outflow tract $(43,44)$. This notion of digenic and/or multigenic rationale to explain differential expressivity and penetrance associated with Mendelian-inherited disease is not novel and is being considered in different forms of glaucomas. It was shown that digenic variants in CYP1B1 and $M Y O C$ contribute to PCG and that variants in both FOXC1 and PITX2 are responsible for some cases of ARS $(10,45)$. This prompted us to explore the frequency of CHD in patients with ARS carrying a Foxc1 mutation and whether or not there is a need to carry on WES to investigate the role of other variants in conjunction with FOXC1 that would explain these cardiac defects. 


\section{Whole Exome Sequencing}

A tool to draw genotype-phenotype correlation out of the 67 FOXC1 variants reported so far to be linked to the ARS, only nine have been shown to be linked to cardiac defects in addition to the ocular defects. A scrutinized review of the literature of these nine variants, namely p.Q70Hfs ${ }^{\star} 8$, p.P79T, p.S82T, p. A85P, p.L86F, p.F112S, p.R127L, p.G149D, and p.R170W, did show that the cardiac phenotype with which they are associated is not as clear as it is presumed. In some cases, the defect is not a structural one, while in most cases, it is found in only one affected child but not in the parent despite sharing the same variant $(33,46)$. This reinforces the notion that another variant from the healthy parent in a genetic and/or molecular pathway implicating FOXC1 would be a potential hit to explain the cardiac-associated phenotype. In the case of p.Q70Hfs* 8 and p.P79T, only one affected patient out of two with the variant has an atrial septal defect (ASD) and PDA, respectively, while the parent carrying the mutated allele has a mild ARS phenotype and no cardiac defects $(47,48)$. The same applies to p.A85P and p.R127L whereby only one of the two affected individuals has either ASD and pulmonary and aortic stenosis or PDA, respectively, whereas the parent from whom the mutation is inherited does not have cardiac defects $(33,49,50)$. In the p.S82T case, the initial description of the familial case did not include any structural cardiac defects (17), whereas the report by Mears et al. mentioned cardiac anomalies (14), and that by $\mathrm{Du}$ et al. mentioned ASD with neither description of the methods nor the number of affected individuals (33). The same confusion applies to the p.G149D missense mutation, whereby the reported mutation was linked to ASD in one patient from a family whose members were not included, and whereby the information on the cardiac defect was only listed in a table. There was no description of the phenotype in the text, nor in the methodology used to assess it (49). As for the p.F112S missense mutation, the two reports documenting this mutation point out to mitral valve regurgitation and/or congestive heart failure as phenotypes encountered at older ages in only two cases with this genotype whereas the rest did not show any cardiac anomaly $(47,51)$. The same applies to p.R170W whereby one patient out of five with the mutation has mitral valve regurgitation requiring its replacement, and another an ASD diagnosed in early adolescent (46). Finally, the p.L86F with only one patient having a myocardial infarct at 41 years of age (52). These facts combined with the phenotypes observed in the Foxc1 initial knockout that did not show any cardiac defects should break down the claims that cardiac structural defects are often associated with the ARS phenotype (24). Indeed, the expression of Foxc1 is barely detected in the mouse developing heart, and only the LacZ harboring knockout construct leads to structural cardiac defects when both Foxc1 alleles are deleted $(47,53)$. The deletion of only one allele of Foxc1 has no effect on heart development in mice even in the context of a genetic model with both Foxc2 alleles deleted $(23,44)$. Cardiac defects are thus seldom associated with the ARS phenotype, and we propose that other genes like NFATC1 when mutated would lead to CHD in the presence of a mutated allele of FOXC1.
The limitations in interpreting the cardiac phenotypes in the anterior segment dysgenesis cases could be therefore solved by applying WES for the parents and siblings of any such indexed patient. In our case, the results even go beyond the anterior segment dysgenesis phenotype to highlight a potential life-threat to members of this family with the expression of a nonsense mutation in DSG2 within its members that could lead to dilated cardiomyopathy and sudden cardiac death.

\section{CONCLUSION}

This is the first study on a familial case of anterior segment dysgenesis glaucoma in Lebanon, a country with still a high rate of consanguineous marriages. We unravel by WES a novel mutation in FOXC1 behind the ocular basic phenotype, and we propose a digenic model for the glaucoma phenotype along a mutation in the DPT gene and another digenic model for CHD involving yet a novel mutation in NFATC1.

\section{ETHICS STATEMENT}

All subjects gave written informed consent in accordance with the Declaration of Helsinki. The protocol-Bioch.GN.01-was approved by the Institution Review Board (IRB), at the American University of Beirut.

\section{AUTHOR CONTRIBUTIONS}

AK: did the experiments, analyzed the data, and wrote the first draft of the paper. CA-H, FB, MK, and MA: did the clinical diagnosis, analyzed the data, and participated in the writing up. $\mathrm{HH}$ and KS: did the recruitment and participated in the analysis of the data. GN: conceived the project, supervised the experiments, analyzed the data, participated the writing up, and obtained the funding (with MA).

\section{ACKNOWLEDGMENTS}

We thank all members of the indexed family for their participation in the study. Special thanks to Mrs. Inaam El-Rassy in the Molecular Core facility for Sanger sequencing. This work was supported by an MPP/URB grant from the American University of Beirut.

\section{FUNDING}

The work was funded by the Medical Practice Plan (MPP) and the University Research Board (URB) at the American University of Beirut (AUB).

\section{SUPPLEMENTARY MATERIAL}

The Supplementary Material for this article can be found online at http://journal.frontiersin.org/article/10.3389/fcvm.2017.00058/ full\#supplementary-material. 


\section{REFERENCES}

1. Leske MC, Heijl A, Hyman L, Bengtsson B, Dong L, Yang Z, et al. Predictors of long-term progression in the early manifest glaucoma trial. Ophthalmology (2007) 114:1965-72. doi:10.1016/j.ophtha.2007.03.016

2. Quigley HA, Broman AT. The number of people with glaucoma worldwide in 2010 and 2020. Br J Ophthalmol (2006) 90:262-7. doi:10.1136/ bjo. 2005.081224

3. Lewis C, Hedberg-Buenz A, DeLuca AP, Stone EM, Alward WLM, Fingert JH. Primary congenital and developmental glaucomas. Hum Mol Genet (2017) 26(R1):R28-R36. doi:10.1093/hmg/ddx205

4. Springelkamp H, Iglesias AI, Mishra A, Hohn R, Wojciechowski R, Khawaja AP, et al. New insights into the genetics of primary open-angle glaucoma based on meta-analyses of intraocular pressure and optic disc characteristics. Hum Mol Genet (2017) 26:438-53. doi:10.1093/hmg/ddw399

5. Liu Y, Allingham RR. Molecular genetics in glaucoma. Exp Eye Res (2011) 93(4):331-9. doi:10.1016/j.exer.2011.08.007

6. Rao KN, Nagireddy S, Chakrabarti S. Complex genetic mechanisms in glaucoma: an overview. Indian JOphthalmol (2011) 59(Suppl):S31-42. doi:10.4103/0301-4738.73685

7. Leske MC, Wu SY, Hennis A, Honkanen R, Nemesure B, Group BES. Risk factors for incident open-angle glaucoma: the Barbados Eye Studies. Ophthalmology (2008) 115:85-93. doi:10.1016/j.ophtha.2007.03.017

8. Leske MC. Open-angle glaucoma - an epidemiologic overview. Ophthalmic Epidemiol (2007) 14:166-72. doi:10.1080/09286580701501931

9. Souzeau E, Tram KH, Witney M, Ruddle JB, Graham SL, Healey PR, et al. Myocilin predictive genetic testing for primary open-angle glaucoma leads to early identification of at-risk individuals. Ophthalmology (2017) 124:303-9. doi:10.1016/j.ophtha.2016.11.011

10. Vincent AL, Billingsley G, Buys Y, Levin AV, Priston M, Trope G, et al. Digenic inheritance of early-onset glaucoma: CYP1B1, a potential modifier gene. Am J Hum Genet (2002) 70:448-60. doi:10.1086/338709

11. Zhou T, Souzeau E, Siggs OM, Landers J, Mills R, Goldberg I, et al. Contribution of mutations in known mendelian glaucoma genes to advanced early-onset primary open-angle glaucoma. Invest Ophthalmol Vis Sci (2017) 58:1537-44. doi:10.1167/iovs.16-21049

12. Girgis N, Chen TC. Genetics of the pediatric glaucomas. Int Ophthalmol Clin (2011) 51:107-17. doi:10.1097/IIO.0b013e31821e538b

13. Ton CC, Hirvonen H, Miwa H, Weil MM, Monaghan P, Jordan T, et al. Positional cloning and characterization of a paired box- and homeoboxcontaining gene from the aniridia region. Cell (1991) 67:1059-74. doi:10.1016/ 0092-8674(91)90284-6

14. Mears AJ, Jordan T, Mirzayans F, Dubois S, Kume T, Parlee M, et al. Mutations of the forkhead/winged-helix gene, FKHL7, in patients with Axenfeld-Rieger anomaly. Am J Hum Genet (1998) 63:1316-28. doi:10.1086/302109

15. Hjalt TA, Semina EV. Current molecular understanding of AxenfeldRieger syndrome. Expert Rev Mol Med (2005) 7:1-17. doi:10.1017/ S1462399405010082

16. Cunningham ET Jr, Eliott D, Miller NR, Maumenee IH, Green WR. Familial Axenfeld-Rieger anomaly, atrial septal defect, and sensorineural hearing loss: a possible new genetic syndrome. Arch Ophthalmol (1998) 116:78-82. doi:10.1001/archopht.116.1.78

17. Gould DB, Mears AJ, Pearce WG, Walter MA. Autosomal dominant AxenfeldRieger anomaly maps to 6p25. Am J Hum Genet (1997) 61:765-8. doi:10.1016/ S0002-9297(07)64340-7

18. Nishimura DY, Swiderski RE, Alward WL, Searby CC, Patil SR, Bennet SR, et al. The forkhead transcription factor gene FKHL7 is responsible for glaucoma phenotypes which map to 6p25. Nat Genet (1998) 19:140-7. doi: $10.1038 / 493$

19. Semina EV, Reiter R, Leysens NJ, Alward WL, Small KW, Datson NA, et al. Cloning and characterization of a novel bicoid-related homeobox transcription factor gene, RIEG, involved in Rieger syndrome. Nat Genet (1996) 14:392-9. doi:10.1038/ng1296-392

20. Zhu H. Forkhead box transcription factors in embryonic heart development and congenital heart disease. Life Sci (2016) 144:194-201. doi:10.1016/j. lfs.2015.12.001

21. Golson ML, Kaestner KH. Fox transcription factors: from development to disease. Development (2016) 143:4558-70. doi:10.1242/dev.112672
22. Lehmann OJ, Sowden JC, Carlsson P, Jordan T, Bhattacharya SS. Fox's in development and disease. Trends Genet (2003) 19:339-44. doi:10.1016/ S0168-9525(03)00111-2

23. Kume T, Jiang H, Topczewska JM, Hogan BL. The murine winged helix transcription factors, Foxc1 and Foxc2, are both required for cardiovascular development and somitogenesis. Genes Dev (2001) 15:2470-82. doi:10.1101/ gad.907301

24. Kume T, Deng KY, Winfrey V, Gould DB, Walter MA, Hogan BL. The forkhead/winged helix gene Mf1 is disrupted in the pleiotropic mouse mutation congenital hydrocephalus. Cell (1998) 93:985-96. doi:10.1016/ S0092-8674(00)81204-0

25. Smith RS, Zabaleta A, Kume T, Savinova OV, Kidson SH, Martin JE, et al. Haploinsufficiency of the transcription factors FOXC1 and FOXC2 results in aberrant ocular development. Hum Mol Genet (2000) 9:1021-32. doi:10.1093/ hmg/9.7.1021

26. Ito YA, Goping IS, Berry F, Walter MA. Dysfunction of the stressresponsive FOXC1 transcription factor contributes to the earlier-onset glaucoma observed in Axenfeld-Rieger syndrome patients. Cell Death Dis (2014) 5:e1069. doi:10.1038/cddis.2014.8

27. Al-Haddad C, Abdulaal M, Badra R, Barikian A, Noureddine B, Farra C. Genotype/phenotype correlation in primary congenital glaucoma patients in the lebanese population: a pilot study. Ophthalmic Genet (2016) 37:31-6. doi: $10.3109 / 13816810.2014 .924015$

28. Souzeau E, Siggs OM, Zhou T, Galanopoulos A, Hodson T, Taranath D, et al. Glaucoma spectrum and age-related prevalence of individuals with FOXC1 and PITX2 variants. Eur J Hum Genet (2017) 25:839-47. doi:10.1038/ ejhg.2017.59

29. Tumer Z, Bach-Holm D. Axenfeld-Rieger syndrome and spectrum of PITX2 and FOXC1 mutations. Eur J Hum Genet (2009) 17:1527-39. doi:10.1038/ ejhg.2009.93

30. Reis LM, Tyler RC, Volkmann Kloss BA, Schilter KF, Levin AV, Lowry RB, et al. PITX2 and FOXC1 spectrum of mutations in ocular syndromes. Eur J Hum Genet (2012) 20:1224-33. doi:10.1038/ejhg.2012.80

31. Komatireddy S, Chakrabarti S, Mandal AK, Reddy AB, Sampath S, Panicker SG, et al. Mutation spectrum of FOXC1 and clinical genetic heterogeneity of Axenfeld-Rieger anomaly in India. Mol Vis (2003) 9:43-8.

32. Kawase C, Kawase K, Taniguchi T, Sugiyama K, Yamamoto T, Kitazawa Y, et al. Screening for mutations of Axenfeld-Rieger syndrome caused by FOXC1 gene in Japanese patients. J Glaucoma (2001) 10:477-82. doi:10.1097/ 00061198-200112000-00007

33. Du RF, Huang H, Fan LL, Li XP, Xia K, Xiang R. A novel mutation of FOXC1 (R127L) in an axenfeld-rieger syndrome family with glaucoma and multiple congenital heart diseases. Ophthalmic Genet (2016) 37:111-5. doi:10.3109/13 816810.2014 .924016

34. Khan AO, Aldahmesh MA, Al-Amri A. Heterozygous FOXC1 mutation $(\mathrm{M} 161 \mathrm{~K})$ associated with congenital glaucoma and aniridia in an infant and a milder phenotype in her mother. Ophthalmic Genet (2008) 29:67-71. doi:10.1080/13816810801908152

35. Takeda U, Utani A, Wu J, Adachi E, Koseki H, Taniguchi M, et al. Targeted disruption of dermatopontin causes abnormal collagen fibrillogenesis. J Invest Dermatol (2002) 119:678-83. doi:10.1046/j.1523-1747.2002.01863.x

36. Tan Y, Iimura K, Sato T, Ura K, Takagi Y. Spatiotemporal expression of the dermatopontin gene in zebrafish Danio rerio. Gene (2013) 516:277-84. doi:10.1016/j.gene.2012.11.074

37. Cooper LJ, Bentley AJ, Nieduszynski IA, Talabani S, Thomson A, Utani A, et al. The role of dermatopontin in the stromal organization of the cornea. Invest Ophthalmol Vis Sci (2006) 47:3303-10. doi:10.1167/iovs.05-1426

38. Marston S. Obscurin variants and inherited cardiomyopathies. Biophys Rev (2017) 9(3):239-43. doi:10.1007/s12551-017-0264-8

39. Marston S, Montgiraud C, Munster AB, Copeland O, Choi O, Dos Remedios C, et al. OBSCN mutations associated with dilated cardiomyopathy and haploinsufficiency. PLoS One (2015) 10:e0138568. doi:10.1371/journal.pone.0138568

40. Abdul-Sater Z, Yehya A, Beresian J, Salem E, Kamar A, Baydoun S, et al. Two heterozygous mutations in NFATC1 in a patient with tricuspid atresia. PLoS One (2012) 7:e49532. doi:10.1371/journal.pone.0049532

41. Ranger AM, Grusby MJ, Hodge MR, Gravallese EM, de la Brousse FC, Hoey T, et al. The transcription factor NF-ATc is essential for cardiac valve formation. Nature (1998) 392:186-90. doi:10.1038/32426 
42. de la Pompa JL, Timmerman LA, Takimoto H, Yoshida H, Elia AJ, Samper E, et al. Role of the NF-ATc transcription factor in morphogenesis of cardiac valves and septum. Nature (1998) 392:182-6. doi:10.1038/32419

43. Lin CY, Lin CJ, Chen CH, Chen RM, Zhou B, Chang CP. The secondary heart field is a new site of calcineurin/Nfatc1 signaling for semilunar valve development. J Mol Cell Cardiol (2012) 52:1096-102. doi:10.1016/j.yjmcc.2012.01.013

44. Seo S, Kume T. Forkhead transcription factors, Foxc1 and Foxc2, are required for the morphogenesis of the cardiac outflow tract. Dev Biol (2006) 296:421-36. doi:10.1016/j.ydbio.2006.06.012

45. Kelberman D, Islam L, Holder SE, Jacques TS, Calvas P, Hennekam RC, et al. Digenic inheritance of mutations in FOXC1 and PITX2: correlating transcription factor function and Axenfeld-Rieger disease severity. Hum Mutat (2011) 32:1144-52. doi:10.1002/humu.21550

46. Gripp KW, Hopkins E, Jenny K, Thacker D, Salvin J. Cardiac anomalies in Axenfeld-Rieger syndrome due to a novel FOXC1 mutation. Am J Med Genet A (2013) 161A:114-9. doi:10.1002/ajmg.a.35697

47. Swiderski RE, Reiter RS, Nishimura DY, Alward WL, Kalenak JW, Searby CS, et al. Expression of the Mfl gene in developing mouse hearts: implication in the development of human congenital heart defects. Dev Dyn (1999) 216:16-27. doi:10.1002/(SICI)1097-0177(199909)216:1<16::AID-DVDY4>3.0.CO;2-1

48. Suzuki T, Takahashi K, Kuwahara S, Wada Y, Abe T, Tamai M. A novel (Pro79Thr) mutation in the FKHL7 gene in a Japanese family with Axenfeld-Rieger syndrome. Am J Ophthalmol (2001) 132:572-5. doi:10.1016/ S0002-9394(01)01059-5

49. Weisschuh N, Dressler P, Schuettauf F, Wolf C, Wissinger B, Gramer E. Novel mutations of FOXC1 and PITX2 in patients with Axenfeld-Rieger malformations. Invest Ophthalmol Vis Sci (2006) 47:3846-52. doi:10.1167/iovs.06-0343
50. Fuse N, Takahashi K, Yokokura S, Nishida K. Novel mutations in the FOXC1 gene in Japanese patients with Axenfeld-Rieger syndrome. Mol Vis (2007) 13:1005-9.

51. Honkanen RA, Nishimura DY, Swiderski RE, Bennett SR, Hong S, Kwon YH, et al. A family with Axenfeld-Rieger syndrome and Peters Anomaly caused by a point mutation (Phe112Ser) in the FOXC1 gene. Am J Ophthalmol (2003) 135:368-75. doi:10.1016/S0002-9394(02)02061-5

52. Saleem RA, Banerjee-Basu S, Berry FB, Baxevanis AD, Walter MA. Structural and functional analyses of disease-causing missense mutations in the forkhead domain of FOXC1. Hum Mol Genet (2003) 12:2993-3005. doi:10.1093/ hmg/ddg 324

53. Winnier GE, Kume T, Deng K, Rogers R, Bundy J, Raines C, et al. Roles for the winged helix transcription factors MF1 and MFH1 in cardiovascular development revealed by nonallelic noncomplementation of null alleles. Dev Biol (1999) 213:418-31. doi:10.1006/dbio.1999.9382

Conflict of Interest Statement: The authors declare that the research was conducted in the absence of any commercial or financial relationships that could be construed as a potential conflict of interest.

Copyright (c) 2017 Khalil, Al-Haddad, Hariri, Shibbani, Bitar, Kurban, Nemer and Arabi. This is an open-access article distributed under the terms of the Creative Commons Attribution License (CC BY). The use, distribution or reproduction in other forums is permitted, provided the original author(s) or licensor are credited and that the original publication in this journal is cited, in accordance with accepted academic practice. No use, distribution or reproduction is permitted which does not comply with these terms. 